\title{
Parâmetros genéticos em progênies de polinização aberta de Enterolobium contortisiliquum (Vell.) Morong em Luiz Antonio, SP, Brasil
}

\author{
Vanessa Zaffani Sant'Ana ${ }^{1}$, Miguel Luiz Menezes Freitas ${ }^{2}$, Mario Luiz Teixeira de Moraes ${ }^{1}$, Marcelo Zanata ${ }^{2}$, \\ Antonio Carlos Scatena Zanatto ${ }^{2}$, Marcela Aparecida de Moraes ${ }^{1}$ e Alexandre Magno Sebbenn ${ }^{2,3}$
}

Recebido: 27.05.2013; aceito: 13.09.2013

\begin{abstract}
Genetic parameters in open-pollinated progenies of Enterolobium contortisiliquum (Vell.) Morong in Luiz Antonio, São Paulo State, Brazil).The aim of this study was to estimate the genetic parameters on a progeny test of Enterolobium contortisiliquum, located at the Fazenda Experimental de Luíz Antônio (IF-SP), São Paulo State, Brazil, for genetic selection and seed production. The coefficients of genetic variation and heritability, as well as the genetic and phenotypic correlations for the silvicultural traits diameter at breast height (DBH) and total height of plants at the age of 19,20 , and 21 years and bifurcation (BIF) and stem straightness (RET), at the age of 19 years were estimated. The $F$ test of analysis of variance detected significant variation among the progeny for the traits $\mathrm{DBH}$, height in the three tested ages, and straightness of the trunk, which indicates that the tested population can be improved by selection among progenies. The estimation of heritability at the level of progeny $\left(h_{m}^{2}\right.$, minimum of 0.50$)$ for all traits was high and at the levels of individual plants $\left(h_{i}^{2}\right.$, minimum 0.18) and within progenies $\left(h_{d}^{2}\right.$, minimum of 0.14$)$ was medium, indicating that the population can be improved by selection among and within progenies. Significant high genetic and phenotypic correlations among pairwise growth traits of the same age were detected as well as among those with different ages. Therefore, the direct selection, of a trait allows indirect selection of another. The results showed the potential of this progeny test to enhance a seed orchard by selection and seed production for commercial and environmental reforestation plans.
\end{abstract}

Key words: genetic and phenotypic correlations, genetic variability, heritability, reforestation, timburí

RESUMO - (Parâmetros genéticos em progênies de polinização aberta de Enterolobium contortisiliquum (Vell.) Morong em Luiz Antonio, SP, Brasil). O objetivo deste estudo foi estimar parâmetros genéticos de teste de progênies de Enterolobium contortisiliquum, implantada na Fazenda Experimental de Luís Antônio (IF-SP), SP, para fins de seleção e produção de sementes. Foram estimados os coeficientes de variação genética e herdabilidade e correlações genéticas e fenotípicas para os caracteres silviculturais, diâmetro a 1,30 m de altura do solo (DAP) e altura total de plantas, aos 19, 20 e 21 anos de idade e para bifurcação (BIF) e retidão do tronco (RET) fuste, aos 19 anos de idade. O teste $F$ das análises de variância detectou variações significativas entre as progênies para os caracteres DAP, altura nas três idades avaliadas e retidão do tronco, o que indica que a média populacional do teste pode ser melhorada pela seleção entre progênies. A estimativa do coeficiente de herdabilidade em nível de progênies $\left(h_{m}^{2}\right.$, mínimo de 0,50$)$ para os caracteres foi alta, e em nível de plantas individuais $\left(h_{i}{ }^{2}\right.$, mínimo de 0,18$)$ e dentro de progênies $\left(h_{d}^{2}\right.$, mínimo de 0,14$)$ foi mediana, indicando que a população pode ser melhorada pela seleção entre e dentro de progênies. Foram também detectadas altas e significativas correlações genéticas e fenotípicas entre os caracteres de crescimento com a mesma idade, e entre aqueles com diferentes idades, portanto a seleção direta em um caráter permite a seleção indireta em outro. Os resultados mostraram o potencial de transformar este teste de progênies em um pomar de sementes para a produção de sementes para reflorestamentos comerciais e ambientais.

Palavras-chave: correlações genética e fenotípica, herdabilidade, reflorestamento, timburí, variabilidade genética

\section{Introdução}

A contínua devastação das florestas brasileiras tem propiciado que grandes riquezas de nossa flora sejam perdidas diariamente. Embora haja grandes movimentos sociais contra essas devastações e existam leis de proteção ambiental, as florestas que abrigam grande diversidade de espécies nativas

1. Universidade Estadual Paulista "Júlio de Mesquita Filho", Avenida Brasil 158, 15385-000 Ilha Solteira, SP, Brasil

2. Instituto Florestal, Rua do Horto 931, 02377-000 São Paulo, SP, Brasil

3. Autor para correspondência: alexandresebbenn@yahoo.com.br 
são destruídas, sem que a maior parte dessas espécies tenham sido estudadas. Dada tais praticas ecologicamente descompromissadas, diariamente são perdidas possibilidades econômicas e sociais que a biodiversidade poderia trazer.

Conhecer a distribuição da variabilidade genética entre e dentro de populações, a dinâmica populacional, a biologia reprodutiva, e aspectos de sua biologia e ecologia é de fundamental importância para a elaboração de estratégias de conservação e restauração de fragmentos. Há, porém, carência de informações sobre as espécies tropicais nativas, o que dificulta a adoção de práticas conservacionistas ou de recuperação de áreas degradadas (Carpi et al. 1996). Estudos fenotípicos e genotípicos para caracteres adaptativos (diâmetro, altura, forma, sobrevivência) são uma opção para quantificar a estrutura genética em populações de uma espécie, utilizando delineamentos experimentais como testes de procedências e progênies (Kageyama \& Dias 1985). É possível manter parte da variabilidade genética das populações em bancos de conservação ex situ de espécies arbóreas em perigo de extinção (Sebbenn \& Ettori 2001). Teste de procedência e progênies também permitem métodos de seleção em programas de melhoramento genético (Sebbenn et al. 1999).

Enterolobium contortisiliquum (Vell.) Morong pertence à família Fabaceae, subfamília Mimosoideae, conhecido pelos nomes populares timbori, tamboril ou orelha-de-macaco. Árvore de grande porte, atingindo altura de 20 a $35 \mathrm{~m}$, encontrada nas florestas pluvial e semidecídua, e particularmente frequente na floresta latifoliada da bacia do Paraná. Sua madeira, leve e macia ao corte, é utilizada para a fabricação de barcos e canoas de tronco inteiro, brinquedos, compensados e caixotaria. Frequentemente é encontrada colonizando áreas desmatadas, em clareiras e bordas de mata. Presta-se à arborização e à recuperação de áreas degradadas, em reflorestamentos mistos principalmente por ter um rápido crescimento inicial, apesar de irregular (Lorenzi 1992, Durigan et al. 2002). As raízes são grossas e longas e servem para jangadas. Contêm porcentagem elevada de saponina na casca e nos frutos, servindo para fabricação de sabão caseiro. É excelente para estudos de reflorestamento e necessita, portanto, de maiores conhecimentos acerca da emergência e do crescimento inicial das mudas nas condições encontradas.

Os objetivos deste estudo foram estimar a variabilidade genética em uma população de
Enterolobium contortisiliquum, para caracteres silviculturais, além de fornecer sementes para plantios de recuperação ambiental. Fornecer subsídios para o melhor entendimento da variação genética dentro de uma população dessa espécie importante, para fins de fomento e conservação genética.

\section{Material e métodos}

Amostragem e Delineamento Experimental - Para instalar este ensaio, em 1989, sementes de polinização aberta foram coletadas de 23 árvores matrizes de uma população natural de Enterolobium contortisiliquum em Bauru, Estado de São Paulo. O experimento foi estabelecido na Estação Experimental de Luiz Antonio, SP, do Instituto Florestal. A E.E. de Luiz Antonio localiza-se nas coordenados $21^{\circ} 40^{\prime} \mathrm{S}$, $47^{\circ} 49^{\prime} \mathrm{W}$, altitude de $550 \mathrm{~m}$ acima do nível do mar. O clima é tropical (Cwa), a temperatura média anual do mês mais quente é de $22,7^{\circ} \mathrm{C}$ e do mês mais frio de $17,2^{\circ} \mathrm{C}$, o inverno é seco, a precipitação média anual é de $1.280 \mathrm{~mm}$ e solo é do tipo latossolo roxo. O teste foi instalado no delineamento experimental de blocos ao acaso, com seis repetições, 23 progênies e cinco plantas por parcela. $\mathrm{O}$ espaçamento utilizado foi o de $3 \times 3 \mathrm{~m}$. Também foi adotada uma bordadura externa de duas linhas da mesma espécie. As mudas foram plantadas em outubro de 1990 . O ensaio foi mensurado aos 19, 20 e 21 anos de idade para o diâmetro a 1,30 m de altura do solo (DAP) (cm) e altura total (h) (m) e aos 19 anos para bifurcação (BIF) e retidão do tronco (RET). Para bifurcação e retidão do tronco, utilizou-se escala de notas, variando de 1 a 5. Para a BIF e RET as descrições das notas utilizadas estão apresentadas nas figuras 1 e 2 , respectivamente.

Análises estatística e estimação de parâmetros - Os teste $F$ das análises de variância foram calculados pelo procedimento GLM e a estimativa de componentes de variância pelo procedimento VARCOMP, utilizando o método de REML (Restricted Maximun Likelihood), ambos procedimentos implementados no programa estatístico SAS (SAS 1999). O possível uso do método REML para estimar os componentes da variância, deve-se ao desbalanceamento experimental em termos do número desigual de árvores sobreviventes por parcelas. Os valores perdidos foram estimados, e os componentes da variância foram ajustados para estes. Para análise de variância e estimativa de componentes de variância, adotou-se o seguinte modelo estatístico: 


$$
Y_{i j k}=m+t_{i}+b_{j}+e_{i j}+d_{i j k}
$$

em que: $Y_{i j k}=$ desempenho médio do $k$-ésimo indivíduo, do $j$-ésimo bloco, da $i$-ésima progênie; $m$ : média geral da variável em análise; $t_{i}=$ efeito aleatório da $i$-ésima progênie $(i=1,2, \ldots, I) ; b_{j}=$ efeito fixo do $j$-ésimo bloco $(j=1,2, \ldots, J) ; e_{i j}=$ efeito da interação entre a $i$-ésima progênie do $j$-ésimo bloco, ou efeito ambiental da $i j$-ésima parcela; $d_{i j k}=$ efeito da $k$-ésimo indivíduo dentro da $i j$-ésima parcela. $K$ é o número de árvores por progênies, $J$ o número de blocos, $I$ o número de progênies e $K$ a média harmônica do número de árvores por parcela.

Das análises de variância foram estimados os componentes: $\sigma_{p}^{2}$ : variância genética entre progênies; $\sigma_{e}^{2}$ : variância devido à interação entre progênies e repetições (ambiental); $\sigma_{d}^{2}$ : variância fenotípica dentro de progênies; $\sigma_{F}^{2}$ : variância fenotípica total $\left(\hat{\sigma}_{F}^{2}=\hat{\sigma}_{p}^{2}+\hat{\sigma}_{e}^{2}+\hat{\sigma}_{d}^{2}\right)$; a variância genética aditiva $\left(\sigma_{A}^{2}\right)$ foi calculada com base na expressão: $\hat{\sigma}_{A}^{2}=\hat{\sigma}_{p}^{2} / \hat{r}_{x p}$, sendo $r_{x y}$ a estimativa do coeficiente de parentesco ou co-variância genética aditiva entre plantas dentro de progênies. Como não existem estimativas do coeficiente de parentesco entre plantas dentro de progênies de polinização aberta para a presente espécie, população e evento reprodutivo, assumiu-se um coeficiente de coancestria $\left(\Theta_{x y}\right)$ de 0,221, conforme estimativa média calculada para diversas espécies arbóreas tropicais polinizadas por animais (Sebbenn 2006), como E. contortisiliquum. Assim, assumindo ausência de endogamia, o coeficiente de parentesco pode ser estimado pela expressão: $r=2 \Theta_{x y}$ e a variância genética aditiva por $\left(\hat{\sigma}_{F}^{2}=\hat{\sigma}_{p}^{2} / 0,442\right.$.

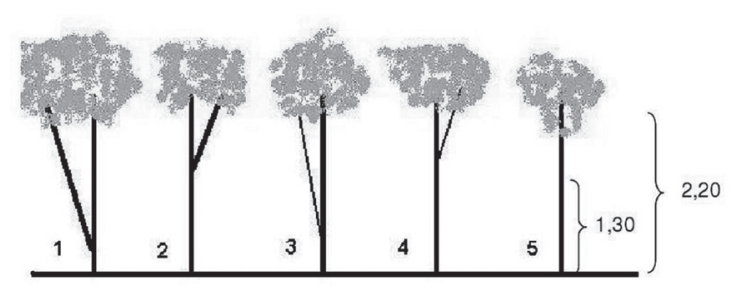

Figura 1. Escala de notas para a bifurcação em relação ao tronco principal em progênies de Enterolobium contortisiliquum.

Figure 1. Grading scale for the bifurcation in relation to the main trunk in progenies of Enterolobium contortisiliquum.

Nota - 1. Bifurcação abaixo de 1,30 m com diâmetro igual ao tronco principal; 2. Bifurcação acima de $1,30 \mathrm{~m}$ com diâmetro igual ao tronco principal; 3. Bifurcação abaixo de $1,30 \mathrm{~m}$ com diâmetro inferior ao tronco principal; 4. Bifurcação acima de $1,30 \mathrm{~m}$ com diâmetro inferior ao tronco principal; 5. Sem bifurcação.
As definições e cálculos dos coeficientes de herdabilidades, coeficientes de variação e medidas de correlações entre caracteres seguiu Namkoong (1979). Os coeficientes de herdabilidade em nível de plantas individuais $\left(h_{i}^{2}\right)$, entre progênies $\left(h_{m}^{2}\right)$, dentro de progênies $\left(h_{d}^{2}\right)$ e coeficiente de variação genética $\left(C V_{g}\right)$ foram estimados por:

$$
\begin{aligned}
& \hat{h}_{i}^{2}=\frac{\hat{\sigma}_{A}^{2}}{\hat{\sigma}_{p}^{2}+\hat{\sigma}_{e}^{2}+\hat{\sigma}_{d}^{2}}, \hat{h}_{m}^{2}=\frac{\hat{\sigma}_{p}^{2}}{\hat{\sigma}_{p}^{2}+\frac{\hat{\sigma}_{e}^{2}}{J}+\frac{\hat{\sigma}_{d}^{2}}{\overline{K J}}}, \hat{h}_{d}^{2}=\frac{\left(1-\hat{r}_{x y}\right) \hat{\sigma}_{A}^{2}}{\hat{\sigma}_{d}^{2}} \mathrm{e} \\
& C V g=\frac{\sqrt{\hat{\sigma}_{p}^{2}}}{\hat{m}} .100
\end{aligned}
$$

Sendo $\hat{m}$ a estimativa da média do caráter.

As correlações fenotípicas $\left(r_{f}\right)$ e genéticas $\left(r_{g}\right)$, entre os caracteres DAP e altura de mesma e diferentes idades foram estimadas a partir dos valores individuais de acordo com as seguintes expressões:

$$
\hat{r}_{f}=\frac{\hat{\sigma}_{F_{X} F_{Y}}}{\sqrt{\hat{\sigma}_{F_{X}}^{2} \cdot \hat{\sigma}_{F_{Y}}^{2}}} \quad \text { e } \quad \hat{r}_{g}=\frac{\hat{\sigma}_{p_{X} p_{Y}}}{\sqrt{\hat{\sigma}_{p_{X}}^{2} \cdot \hat{\sigma}_{p_{Y}}^{2}}}
$$

em que: $\sigma_{F_{x} F_{Y}}$ e $\sigma_{p_{x} p_{y}}$ são os produtos cruzados fenotípicos e genéticos dos caracteres $x$ e $y$, estimados das análises de covariância, $\sigma_{F_{X}}^{2}, \sigma_{p_{X}}^{2}, \sigma_{F_{Y}}^{2}$ e $\sigma_{P^{2}}^{2}$ são as variâncias fenotípicas e genéticas dos caracteres $x$ e $y$, respectivamente (Falconer 1987).

\section{Resultados e Discussão}

As progênies de Enterolobium contortisiliquum foram avaliadas em três anos consecutivos, correspondendo a 19, 20 e 21 anos de idade (tabela 1). O DAP apresentou, em média, valores de 22,07, 22,62 e 23,07 cm aos 19, 20 e 21 anos de idade, respectivamente, o que implica em incremento médio anual (IMA) de mínimo de $1,09 \mathrm{~cm}$ aos 21 anos de idade. A altura total das progênies foi de 13,04, 14,02 e 14,73 m aos 19, 20 e 21 anos de idade, respectivamente, correspondendo ao IMA mínimo de 0,68 m. Pode-se observar que os IMAs para os caracteres de crescimento estudados foram menores com o passar dos anos, o que era esperado, pois é comum espécies arbóreas nativas apresentarem um crescimento rápido na sua fase mais jovem, e esse crescimento diminuir com o passar dos anos. 
As progênies de Enterolobium contortisiliquum apresentaram maior crescimento em comparação a algumas outras espécies arbóreas nativas, como por exemplo: Cariniana legalis $(\mathrm{DAP}=13,5 \mathrm{~cm}$ e ALT $=12,3 \mathrm{~m}$ ) aos 17 anos de idade (Sebbenn et al. 2001); Gallesia integrifolia (DAP $=21,8 \mathrm{~cm} \mathrm{e}$ ALT $=13,6 \mathrm{~m}$ ) aos 20 anos de idade (Freitas et al. 2008); Myracrodruon urundeuva (DAP = 13,1 cm e $\mathrm{ALT}=11,5 \mathrm{~m}$ ) aos 17 anos de idade (Freitas et al. 2007); Astronium fraxinifolium (DAP $=9,4 \mathrm{~cm} \mathrm{e}$ ALT $=8,6 \mathrm{~m})$ aos 14 anos de idade e Terminalia argentea $(\mathrm{DAP}=12,8 \mathrm{~cm}$ e ALT $=9,7 \mathrm{~m})$ aos 14 anos de idade (Otsubo 2011). Portanto, em comparação as espécies citadas, E. contortisiliquum apresenta maior potencial para utilização em reflorestamentos comerciais e ambientais.

As progênies de Enterolobium contortisiliquum apresentaram uma média de 3,44 para a bifurcação do tronco e 1,67 para a retidão do tronco (tabela 1). Isto que corresponde a tortuosidade acentuada em toda a extensão do tronco e a bifurcação abaixo de $1,30 \mathrm{~m}$ de altura do solo, com diâmetro inferior ao tronco principal. Contudo, estes valores médios podem ser melhorados pela seleção de genitores dentro do teste com menor tortuosidade e ausência de bifurcação.

Apresentou alto coeficiente de variação experimental, variando de $14,5 \%$ (BIF) a $36,7 \%$ (DAP), indicando que o controle do ambiente foi relativamente baixo. No entanto, a análise de variância detectou diferenças significantes entre progênies para as variáveis DAP, altura total de plantas e retidão do tronco (tabela 1), o que indica a possibilidade de alterar a média populacional destes valores pela seleção entre e dentro de progênies. Ressalta-se ainda que alta variação experimental $\left(C V_{\text {exp }}\right)$ detectada não interferiu na detecção da variação genética entre as progênies.

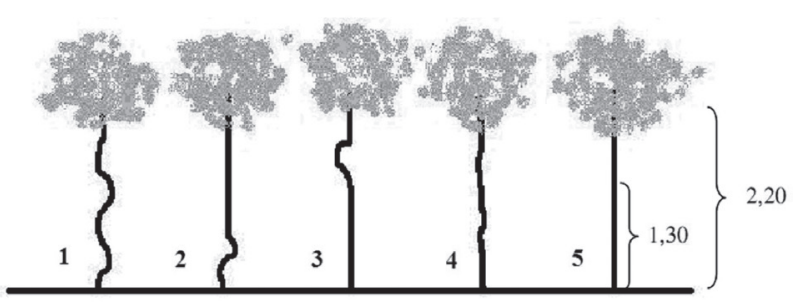

Figura 2. Escala de notas para a retidão do tronco em progênies de Enterolobium contortisiliquum.

Figure 2. Grading scale for the stem straightness in progenies of Enterolobium contortisiliquum.

Nota 2 - 1. Tortuosidade acentuada em toda extensão; 2. Tortuosidade acentuada abaixo de $1,30 \mathrm{~m} ; 3$. Tortuosidade acentuada acima de $1,30 \mathrm{~m}$; 4 . Leve tortuosidade em toda extensão; 5. Sem tortuosidade.
O coeficiente de variação genético foi expressivo para o DAP (mínimo de 10,7\%) e a altura total de plantas (mínimo de 9,2\%), porém foi baixo para a retidão do tronco $(4,2 \%)$, o que reforça a possibilidade de melhorar a média da população para os caracteres de crescimento pela seleção (tabela 2).

A estimativa de coeficientes de herdabilidade permite avaliar se caracteres são herdáveis (Bueno et al. 2001) e com isso melhorar a população por seleção. As estimativas dos coeficientes de herdabilidade médias entre as progênies $\left(h_{m}^{2}\right)$ foram, no mínimo altas, variando de 0,50 (forma do tronco) a 0,67 (ALT 21) e maior para todos os caracteres estudados do que as obtidas para herdabilidade dentro de progênies $\left(h_{d}^{2}\right)$ e em nível de plantas individuais $\left(h_{i}^{2}\right)$ (tabela 2). Isso indica que os caracteres estudados têm forte controle genético em nível de progênie. Pode-se observar também que houve pequena variação nos valores de herdabilidades nos três anos de investigação. Isso pode ser explicado pelo fato de que o coeficiente de herdabilidade pode apresentar variação conforme a idade da planta, pois até que haja completo estabelecimento no campo e equilíbrio, pode haver influência menor ou maior do ambiente na manifestação dos caracteres de crescimento (Ettori et al. 2006). De acordo com Vencovsky \& Barriga (1992), as herdabilidades em nível de média de progênies podem ser superiores às individuais, quando os efeitos ambientais da primeira são minimizados pelo número de repetições e de plantas por parcela. Portanto, a seleção pode ser mais eficiente com base nas médias de progênies do que em plantas individuais.

Os coeficientes de correlação genética foram positivos e significativos para todos os pares de caracteres avaliados (tabela 3). De acordo com Sampaio et al. (1999), a seleção quando é feita em variáveis com correlações positivas e de alta magnitude, espera-se uma alta resposta correlacionada, ou seja, ocorre uma vantagem na seleção que é a mesma para esses caracteres. As maiores correlações encontradas foram entre DAP11 $\times$ DAP10, ALT10 $\times$ DAP10 e ALT11 $\times$ DAP10 com 0,98 . Neste caso pode-se optar por conduzir a seleção com base nas características de ALT e DAP.

As estimativas das correlações fenotípicas evidenciaram também associações positivas e significativas, embora fossem menores que as correlações genéticas. A seleção indireta pode ser mais eficiente nesse caso. As associações positivas entre os caracteres indicam que a seleção em um caráter pode trazer ganhos indiretos em outro, em especial entre DAP aos 19 e 20 anos de idade, que apresentaram as maiores correlações $(0,98)$ (tabela 3$)$. 
Tabela 1. Probabilidades do teste $F$ da análise de variância, estimativas das médias e do coeficiente de variação experimental $\left(C V_{\text {exp }}\right)$ para os caráteres: diâmetro a $1,30 \mathrm{~m}$ de altura do solo (DAP), altura (ALT) e bifurcação (BIF) e retidão do tronco (RET) em 23 progênies de Enterolobium contortisiliquum aos 19, 20 e 21 anos de idade.

Table 1. Probabilities of $F$ test for variance analysis, estimates of the mean and the experimental coefficient of variation $\left(C V_{\text {exp }}\right)$ for the traits: diameter at breast height (DAP), height (ALT), bifurcation (BIF), and stem straightness (RET) for 23 progenies of Enterolobium contortisiliquum at the age of 19, 20, and 21 years.

\begin{tabular}{lcccccccc}
\hline Estimativas & DAP19 $(\mathrm{cm})$ & DAP20 $(\mathrm{cm})$ & DAP21 $(\mathrm{cm})$ & ALT19 $(\mathrm{m})$ & ALT20 $(\mathrm{m})$ & ALT21 $(\mathrm{m})$ & \multicolumn{1}{c}{ BIF } & RET \\
\hline BL & 0,1525 & 0,1725 & 0,3197 & 0,2254 & 0,0107 & 0,2356 & 0,8641 & 0,7844 \\
PROG & $<0,0001$ & $<0,0001$ & 0,0002 & $<0,0001$ & $<0,0001$ & $<0,0001$ & 0,4903 & 0,0012 \\
$C V_{\text {exp }}(\%)$ & 36,67 & 36,47 & 36,34 & 31,39 & 31,39 & 33,10 & 14,52 & 22,31 \\
Média & 22,07 & 22,62 & 23,07 & 13,04 & 14,02 & 14,73 & 3,44 & 1,67 \\
\hline
\end{tabular}

Tabela 2. Estimativas dos coeficientes de herdabilidade e coeficiente de variação genético para os caracteres diâmetro a $1,30 \mathrm{~m}$ de altura do solo (DAP), altura total (ALT) e retidão do tronco (RET) em 23 progênies de Enterolobium contortisiliquum aos 19, 20 e 21 anos de idade, na Estação Experimental de Luiz Antônio, SP, Brasil.

Table 2. Estimates of heritability and genetic variation coefficients for the characters diameter at breast height (DAP), total height (ALT), and stem straightness (RET) of 23 progenies of Enterolobium contortisiliquum at the age of 19, 20, and 21 years in Estação Experimental de Luiz Antônio, São Paulo State, Brazil.

\begin{tabular}{cccccccc}
\hline Parâmetros Genéticos & DAP19 & DAP20 & DAP21 & ALT19 & ALT20 & ALT21 & RET \\
\hline Herdabilidade individual - $\hat{h}_{i}^{2}$ & 0,32 & 0,32 & 0,33 & 0,36 & 0,31 & 0,35 & 0,18 \\
Herdabilidade média de progênies - $\hat{h}_{m}^{2}$ & 0,65 & 0,65 & 0,65 & 0,66 & 0,62 & 0,67 & 0,50 \\
Herdabilidade dentro de progênies - $\hat{h}_{d}^{2}$ & 0,26 & 0,26 & 0,27 & 0,31 & 0,26 & 0,29 & 0,14 \\
Coeficiente de variação genético $(\%)-C V_{g}$ & 10,8 & 10,7 & 10,7 & 10,1 & 9,2 & 10,3 & 4,2 \\
\hline
\end{tabular}

Tabela 3. Correlação genética (diagonal superior) e fenotípicas (diagonal inferior) para os caracteres diâmetro a 1,30 m de altura do solo (DAP) e altura (ALT) em 23 progênies de Enterolobium contortisiliquum aos 19, 20 e 21 anos de idade. ${ }^{* *} p<0,01$.

Table 3. Genetic (upper diagonal) and phenotypic (lower diagonal) correlations for the traits diameter at breast height (DAP) and height (ALT) for 23 progenies of Enterolobium contortisiliquum at the age of 19, 20, and 21 years. ${ }^{* *} p<0.01$.

\begin{tabular}{ccccccc}
\hline Variáveis & DAP19 & DAP20 & DAP21 & ALT19 & ALT20 & ALT21 \\
\hline DAP19 & & $0,89 * *$ & $0,86 * *$ & $0,84 * *$ & $0,87 * *$ & $0,86 * *$ \\
DAP20 & & & $0,98 * *$ & $0,91 * *$ & $0,98 * *$ & $0,98 * *$ \\
DAP21 & & & & $0,90 * *$ & $0,98 * *$ & $1,00 * *$ \\
ALT19 & $0,72 * *$ & $0,74 * *$ & $0,74 * *$ & & $0,88 * *$ & $0,83 * *$ \\
ALT20 & $0,63 * *$ & $0,66 * *$ & $0,66 * *$ & $0,68 * *$ & & $0,85 * *$ \\
ALT21 & $0,75 * *$ & $0,78 * *$ & $0,81 * *$ & $0,91 * *$ & $0,79 * *$ & \\
\hline
\end{tabular}

Os resultados, em termos de conservação genética $e x$ situ, mostraram que existe significativa variação genética entre progênies na população amostrada de Enterolobium contortisiliquum, logo substancial variação genética entre progênies foi retida no banco. Os resultados também mostraram que os caracteres de crescimentos apresentam substancial variação genética e tem forte controle genético entre e dentro de progênies, logo a média da população pode ser melhorada pela seleção de progênies e genótipos superiores dentro de progênies para comporem a população parental, em um pomar de sementes por mudas. A seleção entre e dentro de progênies pode gerar 
genótipos com melhores características em termos de DAP, altura e retidão do tronco. Contudo, como foram testadas apenas 23 progênies, a seleção entre progênies vai reduzir a variabilidade genética e o tamanho efetivo nas sementes coletas de pomar de sementes. Devido a isso, e como as sementes oriundas deste teste podem ser utilizadas para fins de reflorestamentos ambientais, nos quais se deseja o máximo tamanho efetivo para fundar estas novas populações, recomenda-se realizar a seleção dentro de progênies, ou melhor a seleção da melhor planta de cada parcela para os caracteres de crescimento, bifurcação e retidão de tronco. A seleção vai manter as 23 progênies, mas reduzir o parentesco dentro das parcelas, reduzindo a chance de ocorrerem cruzamentos entre parentes dentro das parcelas e, consequentemente, de ocorrer endogamia por cruzamento entre parentes, pois irmãos serão eliminados dentro das parcelas. Em consequência disso, as sementes coletadas em pomar com estas características, além de produzir sementes com o tamanho efetivo representativo destas 23 progênies conservadas ex situ, vai também disseminar parte da variabilidade genética da população original, que deu origem a este teste de progênies, garantindo a sobrevivência de parte dos genes dessa população nas novas populações dos reflorestamentos ambientais originados com as sementes coletadas no proposto pomar.

\section{Agradecimentos}

Ao Conselho Nacional de Pesquisa e Desenvolvimento (CNPq), pela concessão de bolsa de Iniciação Científica à primeira Autora; ao Instituto de Florestal, pela infraestrutura oferecida para o desenvolvimento do trabalho; à técnica de Laboratório Selma B. Moraes, pelo apoio.

\section{Literatura citada}

Bueno, L.C.S., Mendes, A.N.G. \& Carvalho, S.P. 2001. Melhoramento de plantas: princípio e procedimentos. UFLA, Lavras.

Carpi, S.M.F., Barbedo, C.J. \& Marcos-Filho, J. 1996. Condicionamento osmótico de sementes de Cedrela fissilis Vell. Revista Brasileira de Sementes 18: 271-275.

Durigan, G., Figliolia, M.B., Kawabata, M., Garrido, M.A.O. \& Baitello, J.B. 2002. Sementes e mudas de árvores tropicais. 2 ed. Páginas e Letras Editora e Gráfica, São Paulo.
Ettori, L.C., Figliolia, M.B. \& Sato, A.S. 2006. Conservação ex situ dos recursos genéticos de espécies florestais nativas: situação atual no Instituto Florestal. In: A.R. Higa \& L.D. Silva (coord.). Pomar de sementes de espécies florestais nativas. FUPEF do Paraná, Curitiba, pp. 203-225.

Falconer, D.S. 1987. Introdução à genética quantitativa. Universidade Federal de Viçosa, Viçosa.

Freitas, M.L.M., Sebbenn, A.M., Zanatto, C.S. \& Moraes, E. 2007. Formação de pomar de sementes a partir da seleção dentro de teste progênies de Myracrodruon urundeuva. Revista do Instituto Florestal 19: 65-72.

Freitas, M.L.M., Sebbenn, A.M., Zanatto, C.S., Moraes, E. \& Moraes, M.A. 2008. Variação genética para caracteres quantitativos em população de Gallesia integrifolia (Spreng.) Harms Revista do Instituto Florestal 20: 165-173.

Kageyama, P.Y. \& Dias, I.S. 1985. Aplicación de conceptos genéticos a espécies forestales nativas en Brasil. Información sobre Recursos Genéticos Forestales 13: 2-10.

Lorenzi, H. 1992. Árvores brasileiras: manual de identificação e cultivo de plantas arbóreas nativas do Brasil. Editora Plantarum, Nova Odessa.

Namkoong, G. Introduction to quantitative genetics in forestry. Forest Service, Washington.

Otsubo, H.C.B. 2011. Espécies árboreas que ocorrem na região do bolsão sul- mato-grossense e variação genética para caracteres silviculturais em um teste de progênies com três espécies em consórcio. Tese de Doutorado. Universidade Estadual Paulista "Júlio de Mesquita Filho", Ilha Solteira.

S.A.S. 1999. Institute Inc. SAS Procedures Guide. Version 8 (TSMO). SAS Institute Inc., Cary.

Sebbenn, A.M. 2006. Sistemas de reprodução em espécies tropicais e suas implicações para a seleção de árvores matrizes para reflorestamentos ambientais. In: A.R. Higa \& L.D. Silva (coord.). Pomar de sementes de espécies florestais nativas. FUPEF do Paraná, Curitiba, pp. 93-138.

Sebbenn, A.M., Siqueira, A.C.M.F., Vencovski, R. \& Machado, J.A.R. 1999. Interação genótipo $\times$ ambiente na conservação ex situ de Peltophorum dubium, em duas regiões do Estado de São Paulo. Revista do Instituto Florestal 11: 75-89.

Sebbenn, A.M., Coelho, A.S.G., Kageyama, P.Y. \& Zanatto, A.C.S. 2001. Depressão por endogamia em populações de jequitibá-rosa. Revista do Instituto Florestal 13: 61-81.

Sebbenn, A.M., Siqueira, A.C.M.F., Gurgel Garrido, L.M.A. \& Angerami, E.M.R.A. 2001. Variabilidade genética e interação genótipo $\times$ locais em jequitibá-rosa - Cariniana legalis (Mart) O Ktze. Revista do Instituto Florestal 12: 13-23.

Vencovsky, R. \& Barriga, P. 1992. Genética biométrica no fitomelhoramento. Sociedade Brasileira de Genética, Ribeirão Preto. 